\title{
Some Asymptotic Properties of the Solutions of Perturbed Nonlinear Differential Equations (*) (**).
}

\author{
PaUl W. SpIKes - WANDA W. SMtTH (Mississippi State, Ms, U.S.A.)
}

Summary. - The behavior of the solutions of a class of perturbed nonlinear second order differential equations is studied where the perturbation term may be unbounded in each of its arguments. Upper and lower bounds for solutions and a nonoscillation theorem are included in the results.

\section{1. - Introduction.}

The behavior of the solutions of the equation

$$
\left(a(t) x^{\prime}\right)^{\prime}+q(t) f(x)=r\left(t, x, x^{\prime}\right)
$$

has been studied extensively in recent years. Most authors investigating this problem have done so under the assumption that $r\left(t, x, x^{\prime}\right) \equiv 0$, or that either $r\left(t, x, x^{\prime}\right)$ or the quotient $r\left(t, x, x^{\prime}\right) / q(t)$ is small in some sense (cf. [1-5, 9, 10,13, 15-18, 20, 23-26]), while relatively few results have been obtained by placing other conditions on $r$ and $q$ (ef. $[6-8,11,12,14,19,21,22]$ ).

In this paper we study the behavior of the solutions of $(*)$ under the assumptions that there are functions $k$ and $p$, and positive constants $P_{1}$ and $P_{2}$ so that

$$
\left|r\left(t, x, x^{\prime}\right)-k(t)\right| \leqslant p\left(t, x, x^{\prime}\right),
$$

and

$$
P_{1} \leqslant k(t) / q(t) \leqslant P_{2} .
$$

We do not require $k$ and $q$ to be small, nor do we make any explicit requirement that the function $p$ be small in any of $t, x$ and $x^{t}$. Our results generalize a number of the results in [14]. In addition, we obtain a new nonoscillation theorem (Theorem 9) for $(*)$ with $f(x) \equiv x$, which gives rise to an open question concerning such a theorem for $(*)$ when $f(x) \neq \equiv x$.

(*) Entrata in Redazione il 7 aprile 1979 .

(**) Research supported by the Mississippi State University Biological and Physical Sciences Research Institute. 


\section{2. - Continuation and boundedness.}

Consider the equation

$$
\left(a(t) x^{\prime}\right)^{\prime}+q(t) f(x)=r\left(t, x, x^{\prime}\right)
$$

where $a, q:\left[t_{0}, \infty\right) \rightarrow(0, \infty), r:\left[t_{0}, \infty\right) \times R^{2} \rightarrow R, f: R \rightarrow R$, and $a^{\prime}, q^{\prime}, f$ and $r$ are continuous. When convenient we will write (1) as the equivalent system

$$
x^{\prime}=y^{\prime}, \quad y^{\prime}=\left(r(t, x, y)-a^{\prime}(t) y-q(t) f(x)\right) / a(t) .
$$

We will also assume that there exist a nonnegative constant $K$, nonnegative continuous functions $h$ and $g$, and a positive function $k$ of class $C^{\prime}$ such that $F^{\prime}(x)=\int_{0}^{\infty} f(s) d s$
satisfies

$$
F(x)+K \geqslant 0 \quad \text { for all } x
$$

and

$$
|r(t, x, y)-k(t)| \leqslant h(t)(F(x)+K)^{\frac{1}{2}}+g(t)|y| .
$$

For any differentiable function $G$, the notation $G^{\prime}(t)_{+}=\max \left\{G^{\prime}(t), 0\right\}$ and $G^{\prime}(t)_{-}=$ $=\max \left\{-G^{\prime}(t), 0\right\}$ will be used.

Since we will be concerned with the behavior of the solutions of equation (1) and system (2) on intervals of the form $[T, \infty)$, we first state the following continuability theorem which extends [23, Thm. 1].

THEOREM 1. - If (3) and (4) hold, then all solutions of (2) can be defined for all $t \geqslant t_{0}$.

A proof of Theorem 1 can be obtained by making simple modifications in the proof of Theorem 1 in [23], and hence the details will not be include here.

A nontrivial solution $x(t)$ of (1), i.e., $x(t)$ satisfies $\sup \{|x(t)|: t \geqslant T\}>0$ for every $T \geqslant t_{0}$, is said to be oscillatory if it has an unbounded set of zeros, and will be called nonoscillatory otherwise. Equation (1) is said to be nonoseillatory if all its solutions are nonoscillatory.

For our next theorem we will need the conditions

$$
\begin{aligned}
& \int_{t_{0}}^{\infty}\left[(a(s) k(s))_{-}^{\prime} /(a(s) k(s))\right] d s<\infty, \\
& \int_{i_{0}}^{\infty}\left[(q(s) / k(s))_{+}^{\prime} /(q(s) / k(s))\right] d s<\infty,
\end{aligned}
$$




$$
\begin{aligned}
& \int_{t_{0}}^{\infty}\left[h(s) /(a(s) q(s))^{\frac{1}{2}}\right] d s<\infty, \\
& \int_{l_{0}}^{\infty}[g(s) / a(s)] d s<\infty,
\end{aligned}
$$

and that there exist positive constants $A, B, m$ and $j$ so that

$$
[q(t) / k(t)] \geqslant A
$$

and

$$
F(x) \geqslant B|x|^{1+j} \quad \text { for }|x| \geqslant m
$$

THEOREM 2, - If (4)-(10) hold, then all solutions of (1) are bounded.

Proof. - Let $x(t)$ be a solution of (1). Observing that (10) implies (3) we define

Then

$$
W(t)=a(t) y^{2}(t) / 2 k(t)+(q(t) / k(t))(F(x(t))+K) .
$$

$$
W^{\prime}(t)=-(a(t) k(t))^{\prime} y^{2}(t) / 2 k^{2}(t)+(q(t) / k(t))^{\prime}(F(x(t))+K)+y(t) r(t, x(t), y(t)) / k(t),
$$

and, since (4) implies

we obtain

$$
y r(t, x, y) \leqslant h(t)(F(x)+K)^{\frac{1}{1}}|y|+g(t) y^{2}+y k(t),
$$

$$
\begin{aligned}
W^{\prime}(t) \leqslant y^{2}(t)(a(t) k(t))_{-}^{\prime} / 2 k^{2}(t)+ & (F(x(t))+K)(q(t) / k(t))_{+}^{t} \\
& +h(t)|y(t)|\left(F^{\prime}(x(t))+K\right)^{\frac{1}{t}} / k(t)+g(t) y^{2}(t) / k(t)+y(t) .
\end{aligned}
$$

Making use of the inequality

leads to

$$
(a(t) q(t)[F(x(t))+K])^{\frac{1}{1}}|y(t)| / k(t) \leqslant W(t)
$$

where

$$
W^{\prime}(t) \leqslant P(t) W(t)+y(t)
$$

$$
P(t)=(a(t) k(t))_{-}^{\prime} /(a(t) k(t))+(q(t) / k(t))_{+}^{\prime} /(q(t) / k(t))+h(t) /(a(t) q(t))^{\frac{1}{2}}+2 g(t) / a(t) .
$$

Integrating over $\left[t_{0}, t\right]$ yields

$$
a(t) y^{2}(t) / 2 k(t)+q(t)(F(x(t))+K) / k(t)-|x(t)| \leqslant W\left(t_{0}\right)-x\left(t_{0}\right)+\int_{t_{0}}^{t} P(s) W(s) d s .
$$


Next notice that for each $t \geqslant t_{0}$ such that $|x(t)|^{j} \geqslant \max \{2 / A B, m\}$ we have $A B|x(t)|^{j} \geqslant$ $\geqslant 2$, and hence $A B|x(t)|^{j+1} \geqslant 2|x(t)|$. It then follows from (9) and (10) that

so that

$$
A B|x(t)|^{1+j} \leqslant(q(t) / k(t))(F(x(t))+K),
$$

$$
(\tilde{q}(t) / k(t))(F(x(t))+\pi) / 2-|x(t)| \geqslant 0 .
$$

But this implies that

$$
(q(t) / z(t))(F(x(t))+K)-|x(t)| \geqslant(q(t) / k(t))(F(x(t))+K) / 2,
$$

from which we obtain

$$
W(t) / 2 \leqslant W\left(t_{0}\right)-x\left(t_{0}\right)+\int_{t_{0}}^{t} P(s) W(s) d s
$$

for each $t \geqslant t_{0}$ such that $|x(t)|^{i} \geqslant \max \{2 / A B, m\}$.

Hence there exists a constant $E_{1}>0$ so that

$$
W(t) \leqslant E_{1}+\int_{t_{0}}^{t} 2 P(s) W(s) d s
$$

for all $t \geqslant t_{0}$. Then Gronwall's inequality, together with (5)-(8), shows that for $t \geqslant t_{0}$

$$
W(t) \leqslant E_{1} \exp \left(2 \int_{t_{0}}^{t} P(s) d s\right) \leqslant E_{2}
$$

for some constant $E_{2}>0$. Therefore we have, in view of $(9)$, that $F^{\prime}(x(t))+K$ is bounded on $\left[t_{0}, \infty\right)$, and, since (10) implies that $F(x) \rightarrow \infty$ as $|x| \rightarrow \infty$, we see that $x(t)$ is bounded on $\left[t_{0}, \infty\right)$.

As an immediate corollary to the proof of Theorem 2 we have

CoROLLARY 3. - If the hypotheses of Theorem 2 are satisfied and $k(t) / a(t)$ is bounded from above, then every solution $(x(t), y(t))$ of $(2)$ is bounded.

REMarK. - Theorem 2 and Corollary 3 are generalizations of Theorem 1 and Corollary 2 in [14] which were obtained for the equation

$$
x^{\prime \prime}+q(t) f(x)=k(t)
$$

under the more restrictive assumptions that $k^{\prime}(t) \geqslant 0$ and $(q(t) / k(t))^{\prime} \leqslant 0$.

The sharpness of condition (10) in obtaining boundedness of solutions of (1) has already been noted in [14] where it was pointed out that $x(t) \doteq \ln t$ is a solution of 
PAUL W. SpIKES - WANDA W. SMITH: Some asymptotic properties, ete. 121

the equation

$$
x^{\prime \prime}(t)+\tanh x=\left(t^{2}-1\right) /\left(t^{2}+1\right)-1 / t^{2}
$$

In this example $F(x)=\ln (\cosh x)$ satisfies $|x|-\ln 2<F(x) \leqslant|x|$ for all $x$, which shows that (10) is quite sharp.

REMarK. - Notice that (6) implies that the quotient $[q(t) / k(t)]$ is bounded from above on $\left[t_{0}, \infty\right)$. Hence there exists a positive constant $A_{1}$ so that

$$
[q(t) / k(t)] \leqslant A_{1}
$$

for $t \geqslant t_{0}$.

\section{3. - Further asymptotic properties.}

In this section we derive some additional asymptotic properties for the solutions of (1) and prove a nonoscillation theorem for a special case of (1). We begin with properties for the nonoscillatory solutions.

THEOREM 4. - Suppose that (4)-(10) hold and that there exists a constant $\alpha>0$ such that

$$
f(x) \leqslant 1 / A_{1}-2 \alpha \text { for } x<0 \text {. }
$$

If in addition

$$
\left(h(t)+g(t)[k(t) / a(t)]^{\frac{1}{2}}\right) / q(t) \rightarrow 0 \quad \text { as } t \rightarrow \infty
$$

and

$$
\int_{t_{0}}^{\infty} q(u) d s=\infty \quad \text { and } \quad \int_{t_{0}}^{\infty}[1 / a(s)]\left(\int_{t_{0}}^{s} q(u) d u\right) d s=\infty
$$

hold, then every nonoscillatory solution of (1) is eventually positive.

Proof. - Suppose that $x(t)$ is a nonoscillatory solution of $(1)$ such that $x(t)<0$ for $t \geqslant t_{1} \geqslant t_{0}$. Integrating (1) over $\left[t_{2}, t\right], t_{2} \geqslant t_{1}$, yields

$$
a(t) x^{\prime}(t)-a\left(t_{2}\right) x^{\prime}\left(t_{2}\right)=\int_{t_{2}}^{t}(r(s, x(s), y(s))-q(s) f(x(s))) d s .
$$

From (4) $r(t, x(t), y(t)) \geqslant k(t)-h(t)(F(x(t))+K)^{\frac{1}{2}}-g(t)|y(t)|$, so that

$$
a(t) x^{\prime}(t)-a\left(t_{2}\right) x^{\prime}\left(t_{2}\right) \geqslant \int_{t_{2}}^{t} q(s)\left[\left(k(s)-h(s)(F(x(s))+K)^{\frac{1}{2}}-g(s)|y(s)|\right) / q(s)-f(x(s))\right] d s .
$$


It follows from the proof of Theorem 1 that $(F(x(t))+K)^{\frac{1}{2}}$ and $|y(t)|(a(t) / k(t))^{\frac{1}{2}}$ are both bounded on $\left[t_{0}, \infty\right)$, which, together with $(9)^{\prime},(11)$ and (12), implies that tor sufficiently large $t_{2}$

$$
\left(k(t)-h(t)(F(x(t))+K)^{\frac{1}{2}}-g(t)|y(t)|\right) / q(t)-f(x(t)) \geqslant \alpha
$$

for $t \geqslant t_{2}$, and hence

$$
a(t) x^{\prime}(t) \geqslant a\left(t_{2}\right) x^{\prime}\left(t_{2}\right)+\alpha \int_{t_{2}}^{t} q(s) d s
$$

for all suffeiently large $t_{2}$. Thus (13) implies that $a(t) x^{\prime}(t) \rightarrow \infty$ as $t \rightarrow \infty$. Hence there exists $T>t_{2}$ so that $x^{\prime}(t)>0$ for $t \geqslant T$, and therefore

$$
a(t) x^{\prime}(t) \geqslant a(T) x^{\prime}(T)+\alpha \int_{T}^{t} q(s) d s \geqslant a \int_{T}^{t} q(s) d s
$$

for $t \geqslant T$. Dividing by $a(t)$ and integrating, we obtain a contradiction to (13), which completes the proof.

The following lemma, which will be utilized below, applies to all solutions of system (2) and hence to all solutions of (1). Its proof is a matter of strainghtforward computation.

LEMrMa 5. - Let $(x(t), y(t))$ be a solution of $(2)$ on $\left[t_{0}, \infty\right)$ and let $E$ be a positive constant. If $G$ is a function of class $C^{3}$ on $\left[t_{0}, \infty\right), t_{1}$ is any number satisfying $t_{1} \geqslant t_{0}$, and

$\nabla(t)=2 F(x(t)) G(t)+2 E x(t) a(t) y(t) G^{\prime}(t) / q(t)$

then

$$
+\left[(a(t) y(t))^{2} /(a(t) q(t))-2 \int_{t_{1}}^{t}(r(s, x(s), y(s)) y(s) / q(s)) d s\right] G(t)
$$

$$
\begin{aligned}
& V^{\prime}(t)=2[E(x(t))-E x(t) f(x(t))] G^{\prime}(t)+2 E x(t) y(t) a(t)\left(G^{\prime}(t) / q(t)\right)^{\prime} \\
&+a(t) y^{2}(t)\left[(2 E+1) G^{\prime}(t)-(a(t) q(t))^{\prime} G(t) /(a(t) q(t))\right] / q(t) \\
&+2 G^{\prime}(t)\left[E r(t, x(t), y(t)) x(t) / q(t)-\int_{t_{1}}^{t}(r(s, x(s), y(s)) y(s) / q(s)) d s\right]
\end{aligned}
$$

REMarK. - The requirement that $G \in C^{3}\left[t_{0}, \infty\right)$ is obviously stronger than is necessary for obtaining. Lemma 5 , however this is the condition that will be imposed whenever Lemma 5 is used in the remainder of the paper. 
THEOREM 6. - Let the hypotheses of Theorem 2 and Lemma 5 be satisfied with $K=0$ and $E \leqslant 1$. If in addition (12) and (13) hold, and

$$
\begin{gathered}
G(t)>0 \quad \text { and } \quad G^{\prime}(t) \geqslant 0, \\
G(t) \rightarrow \infty \quad \text { and } \quad a(t)\left(G^{\prime}(t) / q(t)\right)^{\prime} / G(t) \rightarrow 0 \quad \text { as } t \rightarrow \infty, \\
(2 E+1) G^{\prime}(t) \leqslant(a(t) q(t))^{\prime} G(t) /(a(t) q(t)), \\
F^{\prime}(x) \leqslant \operatorname{Exf}(x) \quad \text { for all } x,
\end{gathered}
$$

and

$$
\int_{t_{0}}^{\infty}\left|(k(s) / q(s))^{\prime}\right| d s<\infty
$$

then every nonoscillatory solution $x(t)$ of $(1)$ satisfies

$$
\limsup _{t \rightarrow \infty} x(t)<\max \left\{m,(1 / A B)^{1 / j}\right\}
$$

Proof. - Let $x(t)$ be a nonoscillatory solution of $(1)$ and let $(x(t), y(t))$ be the corresponding solution of (2). Notice that (4) holding with $K=0$ implies that $F(x) \geqslant 0$ for all $x$, and hence $x f(x) \geqslant 0$ for all $x$ by (17). Then all hypotheses of Theorem 4 are satisfied, which guarantees the existence of $T_{1} \geqslant t_{0}$ such that if $T \geqslant T_{1}$, then $x(t)>0$ for $t \geqslant T$. Also (14), (16), (17), and Lemma 5 imply that for all $t \geqslant T$

where

$$
V^{\prime}(t) \leqslant 2 E Q(t)+2 G^{\prime}(t)\left[E S_{1}(t)-\int_{T}^{t} S_{2}(s) d s\right]
$$

$$
Q(t)=a(t) x(t) y(t)\left(G^{\prime}(t) / q(t)\right)^{\prime}, \quad S_{1}(t)=r(t, x(t), y(t)) x(t) / q(t)
$$

and

$$
\$_{2}(t)=r(t, x(t), y(t)) y(t) / q(t)
$$

Now suppose $x(t)$ is not eventually monotonic and that $\lim _{t \rightarrow \infty} \sup x(t)>\max \{m$, $\left.(1 / A B)^{1 / j}\right\}$. Then there exists $\lambda>0$ and an increasing sequence $\left\{t_{n}\right\}$ of zeros of $y(t)$ so that $t_{n} \rightarrow \infty$ as $n \rightarrow \infty$, and $x\left(t_{n}\right)>\max \left\{m+\lambda,(1 / A B+\lambda)^{1 / j}\right\}$. Let $\varepsilon=m \lambda B / 4$. From (4) and the fact that $E \leqslant 1$ we have

$$
E q(t) S_{1}(t) \leqslant h(t) x(t)(F(x(t)))^{\frac{2}{2}}+g(t) x(t)|y(t)|+E x(t) k(t)
$$

and

$$
-q(t) S_{2}(t) \leqslant h(t)|y(t)|(F(x(t)))^{\frac{1}{2}}+g(t) y^{2}(t)-y(t) k(t)
$$


124 PAUL W. SPIKES - WANDA W. SMITH: Some asymptotic properties, ete.

Moreover, we have from Theorem 2 that $x(t), F(x(t))$, and $y(t)(a(t) / k(t))^{\frac{1}{2}}$ are all bounded and from (9) that $k(t) / q(t)$ is bounded. Hence there exists a positive constant $L_{1}$ such that

$$
E S_{1}(t) \leqslant L_{1}\left[h(t) / q(t)+g(t) /(a(t) q(t))^{t}\right]+E k(t) x(t) / q(t)
$$

for all $t \geqslant T$. Similarly we have

$$
-S_{2}(t) \leqslant L_{2}\left[h(t) /(a(t) q(t))^{\frac{1}{2}}+g(t) / a(t)\right]-k(t) y(t) / q(t)
$$

for some constant $L_{2}>0$. Thus

$$
-\int_{T}^{t} S_{2}(s) d s \leqslant L_{2} \int_{T}^{t}\left[h(s) /(a(s) q(s))^{\frac{1}{2}}+g(s) /(a(s))\right] d s-\int_{T}^{t}[k(s) y(s) / q(s)] d s,
$$

and an integration by parts gives

$$
\int_{T}^{t}[k(s) y(s) / q(s)] d s=k(t) x(t) / q(t)-k(T) x(T) / q(T)-\int_{T}^{t}(k(s) / q(s))^{\prime} x(s) d s .
$$

Since $E \leqslant 1$ and $x(t)>0$, then

$$
\begin{aligned}
& E S_{1}(t)-\int_{T}^{t} S_{2}(s) d s \leqslant L_{1}\left[h(t) / q(t)+g(t) /(a(t) q(t))^{\frac{1}{2}}\right] \\
& \quad+L_{2} \int_{T}^{t}\left[h(s) /(a(s) q(s))^{\frac{1}{2}}+g(s) / a(s)\right] d s+\int_{T}^{t}\left|(k(s) / q(s))^{\prime}\right| x(s) d s+k(T) x(T) / q(T)
\end{aligned}
$$

for $t \geqslant T$. In view of (7), (8), (9)',(12) and (18), we see that for $T$ sufficiently large

$$
E S_{1}(t)-\int_{T}^{t} S_{2}(s) d s<\varepsilon+k(T) x(T) / q(T),
$$

and therefore

$$
V^{\prime}(t) \leqslant 2 E Q(t)+2[\varepsilon+k(T) x(T) / q(T)] G^{\prime}(t) .
$$

An integration yields

$$
V(t) \leqslant 2 L_{3}+2 E \int_{T}^{t} Q(s) d s+2 G(t)[\varepsilon+k(T) x(T) / q(T)]
$$

for some positive constant $L_{3}$ : Then for each $n>T$

$$
F\left(x\left(t_{n}\right)\right) G\left(t_{n}\right) \leqslant L_{3}+E \int_{T}^{t_{n}} Q(s) d s+G\left(t_{n}\right)[\varepsilon+k(T) x(T) / q(T)]+G\left(t_{n}\right) \int_{T}^{t_{n}} S_{2}(s) d s .
$$


Using (4), (7), (8), and (18) as above we obtain

$$
\int_{T}^{t_{n}} S_{2}(s) d s<\varepsilon+l\left(t_{n}\right) x\left(t_{n}\right) / q\left(t_{n}\right)-k(T) x(T) / q(T) .
$$

Also, an integration by parts gives

$$
\begin{aligned}
2 \int_{T}^{t_{n}} Q(s) d s=a\left(t_{n}\right)\left(G^{\prime}\left(t_{n}\right) / q\left(t_{n}\right)\right)^{\prime} x^{2}\left(t_{n}\right)-a(T)\left(G^{\prime}(T) / q(T)\right)^{\prime} x^{2}(T) & \\
& -\int_{T^{\prime}}^{t_{n}} x^{2}(s)\left[a(s)\left(G^{\prime}(s) / q(s)\right)^{\prime}\right]^{\prime} d s .
\end{aligned}
$$

Observing that

$$
\int_{T}^{t_{n}}\left[a(s)\left(G^{\prime}(s) / q(s)\right)^{\prime}\right]^{\prime} d s=a\left(t_{n}\right)\left(G^{\prime}\left(t_{n}\right) / q\left(t_{n}\right)\right)^{\prime}-a(T)\left(G^{\prime}(T) / q(T)\right)^{\prime}
$$

and that $x(t)$ is bounded, say $x^{2}(t) \leqslant B_{1}$, it follows that there exists a constant $L_{4}>0$ so that

$$
F\left(x\left(t_{n}\right)\right) \leqslant\left(L_{4}+B_{1} a\left(t_{n}\right)\left|\left(G^{\prime}\left(t_{n}\right) / q\left(t_{n}\right)\right)^{\prime}\right|\right) / G\left(t_{n}\right)+2 \varepsilon+k\left(t_{n}\right) x\left(t_{n}\right) / q\left(t_{n}\right) .
$$

Then (15) implies that there exists $N$ such that

$$
F\left(x\left(t_{N}\right)\right) \leqslant 3 \varepsilon+k\left(t_{N}\right) x\left(t_{N}\right) / q\left(t_{N}\right),
$$

and hence by conditions (9) and (10) we have

$$
x^{j}\left(t_{N}\right)<3 \varepsilon / m B+1 / A B<\lambda+1 / A B,
$$

which is a contradiction. This completes the proof for the case when $x(t)$ is not eventually monotonic.

Next suppose that $x(t)$ is eventually monotonic, then

$$
\limsup _{t \rightarrow \infty} x(t)=\operatorname{limit}_{t \rightarrow \infty} x(t)=C .
$$

If $C>\max \left\{m,(1 / A B)^{1 / j}\right\}$ there exist $\lambda>0$ and $T_{1}>t_{0}$ such that $x(t)>\max \{\mathrm{m}$, $\left.(1 / A B+\lambda)^{1 / j}\right\}$ for $t \geqslant T_{1}$ : Also, from the proof of Theorem 2 we have $|y(t)|(a(t) / k(t))^{\frac{1}{3}}$ is bounded, and hence (12) implies that there exists $T \geqslant T_{1}$ so that

$$
(h(t) F(x(t))+g(t)|y(t)|) / q(t)<\lambda B / 2
$$


for $t \geqslant T$. Now integrate (1) to obtain

$$
a(t) y(t)-a(T) y(T)=\int_{T}^{t}[r(s, x(s), y(s))-q(s) f(x(s))] d s .
$$

But (10) and (17) imply that

$$
B x^{i \div 1}(t) \leqslant F(x(t)) \leqslant E x(t) f(x(t)) \leqslant x(t) f(x(t))
$$

so that

$$
f(x(t)) \geqslant B x^{j}(t)>1 / A+\lambda B
$$

for $t \geqslant T$. Then from (4), (9), and (12) we have

$$
\begin{aligned}
r(t, x(t), y(t))-q(t) f(x(t)) \leqslant h(t) & F(x(t))+g(t)|y(t)|-q(t)(1 / A+\lambda \beta)+k(t) \\
& \leqslant[(h(t) F(x(t))+g(t)|y(t)|) / q(t)-\lambda B] q(t)<-\lambda B q(t) / 2
\end{aligned}
$$

for $t \geqslant T$. Hence

$$
a(t) y(t)<a(T) y(T)-(\lambda B / 2) \int_{T}^{t} q(s) d s
$$

and therefore it follows from (13) that $y(T)<0$ for sufficiently large $T$. Upon dividing by $a(t)$ and integrating we see from (13) that $y(t) \rightarrow-\infty$ as $t \rightarrow \infty$. But this is impossible since $x(t)>0$. This completes the proof of the theorem.

The equation

$$
x^{\prime \prime}+\left(t^{4}+\sin t\right) x^{3}=t^{\frac{1}{3}} x^{2} \cos x+4 t^{4}, \quad t \geqslant 2,
$$

satisfies all the hypotheses of Theorem 6 with $q(t)=t^{4}+\sin t, k(t)=4 t^{4}, h(t)=2 t^{\frac{1}{2}}$, $a(t) \equiv 1, g(t) \equiv 0, G(t)=\left(t^{4}+\sin t\right)^{\frac{1}{2}}, m=0, j=3, E=1, B=\frac{1}{4}$ and $A=15 / 64$. Thus if $x(t)$ is a nonoscillatory solution of $(19)$, then $\lim _{t \rightarrow \infty} \sup x(t) \leqslant(256 / 15)^{\frac{b}{3}}<3$. Also observe that the term $t^{\frac{1}{2}} x^{2} \cos x$ in the right member of (19) is unbounded in both $t$ and $x$.

REMark. - Notice that in the proof of Theorem 6 that neither Lemma 5, conditions (14)-(16), nor condition (18) was used for the case when the solution of (1) was eventually monotonic.

Our next two results describe the behavior of the oscillatory solutions of (1).

THEOREM 7. - If conditions (12), (14)-(18) and the hypotheses of Theorem 2 and Lemma 5 hold with $K=0$ and $E=1$, then every oscillatory solution $x(t)$ of $(1)$ satisfies $\liminf _{t \rightarrow \infty} x(t)=0$ and $\limsup _{t \rightarrow \infty} x(t) \leqslant \max \left\{m,(1 / A B)^{1 / i}\right\}$. 
PaUL W. SpIKES - WANDA W. SMrTH: Some asymptotic properties, eto. 127

Proof. - If $x(t)$ is an oscillatory solution of (1), then by an argument similar to that used in the proof of Theorem 6 we arrive at the inequality

$$
V^{\prime}(t) \leqslant 2 Q(t)+2 G^{\prime}(t)\left[S_{1}(t)-\int_{T}^{t} S_{2}(s) d s\right]
$$

for every $t \geqslant T$, where $T$ is any number satisfying $T \geqslant t_{0}$.

Now assume that $\liminf _{t \rightarrow \infty} x(t)=-2 \lambda, \lambda>0$. From $(9)^{\prime}$ we have $k(t) / q(t) \geqslant B_{2}=$ $=1 / A_{1}$. Let $\varepsilon=\lambda B_{2} / 4$, and proceeding as in the proof of Theorem 6 we obtain

$$
\dot{V}^{\prime}(t) \leqslant 2 Q(t)+2[\varepsilon+k(T) x(T) / q(T)] G^{\prime}(t)
$$

for $T$ sufficiently large. Since $\lim _{t \rightarrow \infty} \inf x(t)=-2 \lambda$ there exists an increasing sequence $\left\{u_{n}\right\}$ of zeros of $y(t)$ such that $u_{1}>T, u_{n} \rightarrow \infty$ as $n \rightarrow \infty$, and $x\left(u_{n}\right)<-\lambda$ for $n=1$, $2, \ldots$ Then integrating we have

$$
F^{\prime}\left(x ( u _ { n } ) \left(G\left(u_{n}\right) \leqslant L_{5}+\int_{T}^{u_{n}} Q(s) d s+G\left(u_{n}\right) \int_{T}^{u_{n}} S_{2}(s) d s+\varepsilon G\left(u_{n}\right)+[k(T) x(T) / q(T)] G\left(u_{n}\right)\right.\right.
$$

for some positive constant $L_{5}$, and repeating the same argument as used in Theorem 6 yields

$$
F\left(x\left(u_{n}\right)\right) \leqslant\left[L_{6}+B_{1} a\left(u_{n}\right)\left|\left(G^{\prime}\left(u_{n}\right) / q\left(u_{n}\right)\right)^{\prime}\right|\right] / G\left(u_{n}\right)+2 \varepsilon+k\left(u_{n}\right) x\left(u_{n}\right) / q\left(u_{n}\right)
$$

where $L_{6}>0$ is a constant. But

$$
2 \varepsilon+k\left(u_{n}\right) x\left(u_{n}\right) / q\left(u_{n}\right)<\lambda B_{2} / 2-\lambda B_{2}=-\lambda B_{2} / 2
$$

and $F\left(x\left(u_{n}\right)\right)>0$ by $(10)$, which is impossible in view of $(15)$. Hence $\liminf _{t \rightarrow \infty} x(t)=0$.

The proof that $\limsup _{t \rightarrow \infty} x(t) \leqslant \max \left\{m,(1 / A B)^{1 / j}\right\}$ (except for minor changes) is the same as the first part of the proof of Theorem 6.

REMARK. - Observe that if $(k(t) / q(t))^{\prime} \geqslant 0$ and $(k(t) / q(t))$ is bounded, then (18) holds. Thus Theorem 7 generalizes Theorem 4 and the first conclusion of Theorem 3 in [14] since these two theorems were proved for the special case of (1),

$$
x^{\prime \prime}+q(t) f(x)=k(t)
$$

with their hypotheses including the requirements that $(k(t) / q(t))^{\prime} \geqslant 0$ and $f^{\prime}(x) \geqslant 0$. Equation (19) is a specific example of an equation satisfying all the hypotheses of Theorem 7 without $(k(t) / q(t))^{\prime}=4 t^{3}(4 \sin t-t \cos t) /\left(t^{4}+\sin t\right)^{2}$ being eventually non-negative. 
By adding two additional conditions to the hypotheses of Theorem 7, we generalize the second conclusion of [14, Thm. 3].

THEOREM 8. - Suppose that the hypotheses of Theorem 7 are satisfied, and that

$$
(a(t) / k(t)) \searrow 0 \quad \text { as } t \rightarrow \infty
$$

and

$$
a^{\prime}(t) /(a(t) k(t))^{\frac{1}{2}} \rightarrow 0 \quad \text { as } t \rightarrow \infty
$$

If $x(t)$ is an oscillatory solution of (1) and $\varepsilon$ is any positive number, then there exists $T \geqslant t_{0}$ such that every interval $[c, d]$ with $e \geqslant T, x(c)=x(d)=0$, and $x(t)<0$ on $(c, d)$ satisfies $d-e<\varepsilon$.

Proor. - Let $x(t)$ be an oscillatory solution of (1) and $\varepsilon>0$ be given. If $t_{1} \geqslant t_{0}$ is any zero of $x(t)$, then two integrations of the second equation in (2) over $\left[t_{1}, t\right]$ gives

$$
x(t)=y\left(t_{1}\right)\left(t-t_{1}\right)+\int_{t_{1}}^{t}(t-s)\left[\left(r(s, x(s), y(s))-a^{\prime}(s) y(s)-q(s) f(x(s))\right) / a(s)\right] d s .
$$

From (4) we have

$$
\begin{aligned}
r(t, x(t), y(t))-a^{\prime}(t) y(t) \geqslant & -h(t)(F(x(t)))^{\frac{1}{2}}-g(t)|y(t)|+k(t)-a^{\prime}(t) y(t) \\
& =k(t)\left(1-h(t)\left(F^{\prime}(x(t))\right)\right)^{\frac{1}{2}} / k(t)-|y(t)|(a(t) / k(t))^{\frac{1}{2}} g(t) /(a(t) k(t))^{\frac{1}{2}} \\
& \left.-y(t)(a(t) / k(t))^{\frac{1}{2}}\left[a^{\prime}(t) /(a(t) k(t))^{\frac{1}{2}}\right]\right) .
\end{aligned}
$$

Also, (6) ensures that $q(t) / k(t)$ is bounded from above, which, together with (12), (21), and the fact that both $F(x(t))$ and $|y(t)|(a(t) / k(t))^{\frac{1}{2}}$ are bounded, implies that for $t_{1}$ sufficiently large

$$
r(t, x(t), y(t))-a^{\prime}(t) y(t) \geqslant k(t) / 2
$$

for all $t \geqslant t_{1}$. Therefore for a suitably chosen $t_{1}$

$$
x(t) \geqslant y\left(t_{1}\right)\left(t-t_{1}\right)+\int_{i_{1}}^{t}(t-s)[(k(s) / 2-q(s) f(x(s))) / a(s)] d s .
$$

Since $|y(t)|(a(t) / k(t))^{\frac{1}{2}}$ is bounded, (20) implies that there exists $T \geqslant t_{1}$ so that

$$
4|y(t)| a(t) / k(t)<\varepsilon \quad \text { for all } t \geqslant T \text {. }
$$


Hence, if $[c, d]$ is any interval satisfying the hypotheses of the theorem, it follows from (17) and the fact that (3) holds with $K=0$ that $f(x(t)) \leqslant 0$ on $[c, d]$, and therefore

$$
0 \geqslant y(c)(d-c)+\int_{e}^{d}[(d-s) k(s) / 2 a(s)] d s .
$$

We then have from (20) and the last inequality that

$$
0 \geqslant y(c)(d-c)+k(c)(d-c)^{2} / 4 a(c)
$$

which in turn implies

$$
d-c \leqslant 4|y(c)|(a(c) / k(c))<\varepsilon .
$$

REMARK. - Observe that the hypotheses of Theorem 8 are also satisfied by (19). Thus all the foregoing results in this paper apply to equation (19), while none of the results in [14] apply to this example.

We now consider the special case of $(\mathbf{1})$ when $f(x) \equiv x$ which we will denote by

$$
\left(a(t) x^{\prime}\right)^{\prime}+q(t) x=r\left(t, x, x^{\prime}\right) .
$$

THeORem 9. - Let the hypotheses of Theorem 7 hold with $f(x) \equiv x$ and $E=\frac{1}{2}$. If, in addition to (13),

$$
\left|\left(a(t)[k(t) / q(t)]^{\prime}\right)^{\prime}\right| / q(t) \rightarrow 0 \quad \text { as } t \rightarrow \infty,
$$

and

$$
\int_{t_{0}}^{\infty}\left[\left|\left(a(s)[k(s) / q(s)]^{\prime}\right)^{\prime}\right| /(a(s) q(s))^{\frac{1}{1}}\right] d s<\infty
$$

hold, then every solution $x(t)$ of $(22)$ satisfies $x(t) \rightarrow(k(t) / q(t))$ as $t \rightarrow \infty$.

Proof. - For any solution $x(t)$ of $(22)$ we have

$$
(a(t) y(t))^{\prime}+q(t)[x(t)-k(t) / q(t)]=r(t, x(t), y(t))-k(t) .
$$

If we define $z(t)=x(t)-k(t) / q(t)$, then $z(t)$ is a solution of the equation

$$
\left(a(t) w^{\prime}\right)^{\prime}+q(t) w=H(t)
$$

where $H(t)=r(t, x(t), y(t))-k(t)-\left(a(t)[k(t) / q(t)]^{\prime}\right)^{\prime}$.

First suppose that $z^{\prime}(t)$ has an unbounded set of zeros. Applying Lemma 5 to equation (25) yields

$$
\begin{aligned}
& V(t)=z^{2}(t) G(t)+z(t) a(t) z^{\prime}(t) Q^{\prime}(t) / q(t) \\
& +\left[\left[a(t) z^{\prime}(t)\right]^{2} /(a(t) q(t))-2 \int_{t_{1}}^{t}(H(s) / q(s)) z^{\prime}(s) d s\right] G(t)
\end{aligned}
$$


and

$V^{\prime}(t)=z(t) z^{\prime}(t) a(t)\left(G^{\prime}(t) / q(t)\right)^{\prime}+a(t)\left[z^{\prime}(t)\right]^{2}\left[2 G^{\prime}(t)-G(t)(a(t) q(t))^{\prime} /(a(t) q(t))\right] / q(t)$

and from (16) we have

$$
+G^{\prime}(t)\left[H(t) z(t) / q(t)-2 \int_{i_{1}}^{t}(H(s) / q(s)) z^{\prime}(s) d s\right]
$$

$$
V^{\prime}(t) \leqslant Q_{1}(t)+G^{\prime}(t)\left[S_{3}(t)+2 \int_{i_{1}}^{t} S_{4}(s) d s\right]
$$

for all $t \geqslant t_{1} \geqslant t_{0}$, where $S_{3}(t)=|H(t) z(t) / q(t)|$,

$$
S_{4}(t)=\left|(H(t) / q(t)) z^{\prime}(t)\right| \quad \text { and } \quad Q_{\mathbf{1}}(t)=z(t) z^{\prime}(t) a(t)\left(G^{\prime}(t) / q(t)\right)^{\prime}
$$

Now let $H_{1}(t)=\left|\left(a(t)[k(t) / q(t)]^{\prime}\right)^{\prime}\right|$, then from (4)

$$
|H(t)| \leqslant|r(t, x(t), y(t))-k(t)|+H_{1}(t) \leqslant h(t)|x(t)|+g(t)|y(t)|+H_{1}(t)
$$

and

$$
\left|H(t) z^{\prime}(t)\right| \leqslant\left[h(t)|x(t)|+g(t)|y(t)|+H_{1}(t)\right]\left[|y(t)|+\left|(k(t) / q(t))^{\prime}\right|\right]
$$

Also, since the hypotheses of Theorem 2 are satisfied we have $x(t), k(t) / q(t), a(t)$. $\cdot y^{2}(t) / k(t)$, and $z(t)$ are all bounded on $\left[t_{0}, \infty\right)$. It then follows from (12) and (23) that $S_{3}(t) \rightarrow 0$ as $t \rightarrow \infty$. By a similar argument, (7), (8), (18) and (24) imply that $\int_{t_{0}}^{\infty} S_{3}(s) d s<\infty$. If $z(t)$ does not converge to zero as $t \rightarrow \infty$, then there exists $\lambda>0$ such that $\limsup _{t \rightarrow \infty}|z(t)|=2 \lambda$. Choose $T \geqslant t_{0}$ so that $S_{3}(t)+\int_{T}^{\infty} S_{4}(s) d s<\lambda^{2} / 8$, then

$$
V^{\prime}(t) \leqslant Q_{1}(t)+\lambda^{2} G^{\prime}(t) / 4
$$

for $t \geqslant T$. Moreover there is an increasing sequence $\left\{t_{n}\right\}$ of zeros of $z^{\prime}(t)$ satisfying $t_{1}>T, t_{n} \rightarrow \infty$ as $n \rightarrow \infty$ and $\left|z\left(t_{n}\right)\right|>\lambda$ for $n=1,2, \ldots$. Then integrating $V^{\prime}(t)$ over $\left[T, t_{n}\right]$ gives

and therefore

$$
V\left(t_{n}\right) \leqslant V(T)+\int_{\infty}^{t_{n}} Q_{1}(s) d s+\lambda^{2} G\left(t_{n}\right) / 4
$$

$$
z^{2}\left(t_{n}\right) G\left(t_{n}\right) \leqslant V(T)+\int_{T}^{t_{n}} Q_{1}(s) d s+\lambda^{2} G\left(t_{n}\right) / 4+2 G\left(t_{n}\right) \int_{T}^{t_{n}} S_{4}(s) d s .
$$

But integrating by parts we have

$$
\int_{T}^{t_{n}} Q_{1}(s) d s \leqslant G_{1}+z^{2}\left(t_{n}\right) a\left(t_{n}\right)\left(G^{\prime}\left(t_{n}\right) / q\left(t_{n}\right)\right)^{\prime} / 2-\int_{T}^{t_{n}}\left(z^{2}(s)\left[\left(G^{\prime}(s) / q(s)\right)^{\prime}\right]^{\prime} / 2\right) d s .
$$


for some constant $C_{1}$. Since $z(t)$ is bounded there is a constant $C$ such that $z^{2}(t)<C$. Arguing as in the proof of Theorem 6 shows that there is a constant $C_{2}$ such that

$$
\int_{T}^{t_{n}} Q_{1}(s) d s \leqslant C_{2}+C a\left(t_{n}\right)\left|\left(G^{\prime}\left(t_{n}\right) / q\left(t_{n}\right)\right)^{\prime}\right|
$$

for all $n=1,2, \ldots$ Thus

$$
z^{2}\left(t_{n}\right) \leqslant\left[C_{3}+C a\left(t_{n}\right)\left|\left(G^{\prime}\left(t_{n}\right) / q\left(t_{n}\right)\right)^{\prime}\right|\right] / G\left(t_{n}\right)+\lambda^{2} / 2
$$

where $C_{3}$ is a positive constant. This is clearly a contradiction in view of (15). Thus we have $z(t) \rightarrow 0$ as $t \rightarrow \infty$ in case $z(t)$ is not eventually monotonic.

If $z(t)$ is eventually monotonic, then $z(t)$ is nonoscillatory and tends to a finite limit as $t \rightarrow \infty$. Suppose that $z(t) \rightarrow 4 \lambda$ as $t \rightarrow \infty$. If $\lambda>0$, then there exists $T_{1}>t_{0}$ so that $z(t)>2 \lambda$. for $t \geqslant T_{1}$. We also have $H(t) / q(t) \rightarrow 0$ as $t \rightarrow \infty$, so we may assume that $T_{1}$ is such that $|H(t)| / q(t)<\lambda$ for $t \geqslant T_{1}$. Then an integration of (25) gives

$$
a(t) z^{\prime}(t)-a\left(T_{1}\right) z^{\prime}\left(T_{1}\right)=\int_{T_{1}}^{t}[H(s)-q(s) z(s)] d s \leqslant \int_{T_{1}}^{t} q(s)[H(s) / q(s)-2 \lambda] d s \leqslant-\lambda \int_{T_{1}}^{t} q(s) d s
$$

and (13) implies that $z^{\prime}(t)$ is eventually negative. Thus for sufficiently large $T \geqslant T_{1}$ we have

$$
z^{\prime}(t) \leqslant-(\lambda / a(t)) \int_{T^{*}}^{t} q(s) d s
$$

and integrating we obtain a contradiction to (13). A similar argument shows that $\lambda<0$ also leads to a contradiction, and hence we conclude that $z(t) \rightarrow 0$ in this case also. This completes the proof of the theorem.

If $x^{2}$ and $x^{3}$ are replaced by $x /\left(1+x^{2}\right)$ and $x$ respectively in (19), the resulting equation

$$
x^{\prime \prime}+\left(t^{4}+\sin t\right) x=t^{\frac{1}{2}} x /\left(1+x^{2}\right)+4 t^{4}, \quad t \geqslant 2,
$$

satisfies all the hypotheses of Theorem 9. Hence every solution $x(t)$ of $(19)^{\prime}$ is such that $x(t) \rightarrow 4$ as $t \rightarrow \infty$.

\section{4. - Concluding remarks.}

Notice that in order to have conditions (14)-(16) satisfied it is necessary that $a(t) q(t) \rightarrow \infty$ as $t \rightarrow \infty$. That some such minimum growth condition is essential 
for obtaining the conclusions of Theorems 7-9 is illustrated by the simple example

$$
x^{\prime \prime}+x=1
$$

which has the solution set $\left\{N_{1} \sin t+N_{2} \cos t+1\right\}$ where $N_{1}$ and $N_{2}$ are arbitrary constants. Here $a(t) \equiv q(t) \equiv 1$, and clearly the conclusions of Theorems 7-9 do not hold.

To further point up the roles played by the functions $q$ and $r$ in equation (1) (and their relationship to one another) in obtaining the conclusions of Theorems 7-9, consider the two examples

$$
x^{n}+4 t^{2} x=x^{\prime} / t+8 t^{2}, \quad t>1,
$$

and

$$
x^{\prime \prime}+4 t^{2} x=8 t^{2}-6 \sin t^{2}, \quad t>1
$$

which both have the oscillatory solution $x(t)=2+3 \cos t^{2}$. Thus the conclusions of none of Theorems 7-9 hold for these examples. In both cases $a(t) \equiv 1, B=\frac{1}{2}, j=1$, and $q(t)=4 t^{2}$. For $(26), r\left(t, x, x^{\prime}\right)=x^{t} g(t)+k(t)$ where $g(t)=1 / t$ and $k(t)=8 t^{2}$; and for (27), $r\left(t, x, x^{\prime}\right)=k_{1}(t)=8 t^{2}-6 \sin t^{2}$. In (26) condition (8) does not hold, while in (27) the difficulty is with conditions (6) and (18). Observe that $q, k$, and $k_{1}$ are all $O\left(t^{2}\right)$, and that both $k(t) / q(t)$ and $k_{1}(t) / q(t)$ tend to 2 as $t \rightarrow \infty$.

As a final remark we point out that it is still an open question as to whether or not a theorem analogous to Theorem 9 can be obtained for equation (1) when $f(x) \neq x$.

\section{REFE RENCES}

[1] F. V. Atkinson, On second order non-linear oscillations, Pacific J. Math., 5 (1955), pp. $643-647$.

[2] J. W. BAKER, On the continuation and boundedness of solutions of a nonlinear differential equation, J. Math. Anal. Appl., 55 (1976), pp. 644-652.

[3] J. W. BAKER, Stability properties of a second order damped and forced nonlinear differential equation, SIAM J. Appl. Math., 27 (1974), pp. 159-166.

[4] T. A. BURton - R. C. Grimmer, On the asymptotic behavior of solutions of $x^{\prime \prime}+$ $+a(t) f(x)=e(t)$, Pacific J. Math., 41 (1972), pp. 43-55.

[5] 'T. A. Burton - R. C. Grimmer, Stability properties of $\left(r(t) u^{\prime}\right)^{\prime}+a(t) f(u) g\left(u^{\prime}\right)=0$, Monatśs. Math., 74 (1970), pp. 211-222.

[6] LU-SAN CHEN, Sufficient conditions for nonoscillation of n-th order nonlinear differential equations, Atti Accad. Naz. Lincei Rend. Cl. Sci. Fis. Mat. Natur., 60 (1976), pp. 27-31.

[7] J. R. GRAEF, A comparison and oscillation result for second order nonlinear differential equations, Abh. Math. Sem. Uni. Hamburg, 49 (1979), pp. 74-76.

[8] J. R. GRAEF, Oscillation, nonoscillation, and growth of solutions of nonlinear functional differential equations of arbitrary order, J, Math. Anal. Appl., 60 (1977), pp. 398-409. 
[9] J. R. GRAeF - P. W. SpIKes, Asymptotic properties of solutions of a second order nonlinear differential equation, Publ. Math. Debrecen, 24 (1977), pp. 39-51.

[10] J. R. GRAEF - P. W. SPIKEs, Asymptotic behavior of solutions of a second order nonlinear differential equation, J. Differential Equations, 17 (1975), pp. 461-476.

[11] J. R. GRAEF - P. W. SpIKES, Sufficient conditions for nonoscillation of a second order nonlinear differential equation, Proc. Amer. Math. Soc., 50 (1975), pp. 289-292.

[12] J. R. GRAEF - P. W. SPIKES, Sufficient conditions for the equation $\left(a(t) x^{\prime}\right)^{\prime}+h\left(t, x, x^{\prime}\right)+$ $+q(t) f\left(x x^{\prime}\right)=e\left(t, x, x^{\prime}\right)$ to be nonoscillatory, Funkeial. Ekvac., 18 (1975), pp. 35-40.

[13] J. R. Graef - P. W. SPIKES, Continuability, boundedness, and asymptotic behavior of solutions of $x^{\prime \prime}+q(t) f(x)=r(t)$, Ann. Mat. Pura Appl., (4) 101 (1974), pp. 307-320.

[14] J. R. Grafi - P. W. SPIKEs, On the behavior of solutions of $x^{\prime \prime}+q(t) f(x)=r(t)$, Atti Accad. Naz. Lincei Rend. Cl. Sci. Fis. Mat. Natur., 54 (1973), pp. 544-550.

[15] R. GRIMmer, On nonoscillatory solutions of a nonlinear differential equation, Proc. Amer. Math. Soc., 34 (1972), pp. 118-120.

[16] M. E. HАMmetr, Nonoscillation properties of a nonlinear differential equation, Proc. Amer. Math. Soc., 30 (1971), pp. 92-96.

[17] L. Hatvani, On the asymptotic behavior of the solutions of $\left(p(t) x^{\prime}\right)^{\prime}+q(t) f(x)=0$, Publ. Math. Debrecen, 19 (1972), pp. 225-237.

[18] A. G. KaRTSatos, On positive solutions of perturbed nonlinear differential equations, J. Math. Anal. Appl., 47 (1974), pp. 58-68.

[19] M. S. KEENER, On the solutions of a certain linear nonhomogeneous second-order differential equation, Applicable Analysis, 1 (1971), pp. 57-63.

[20] T. Kusano - H. Onose, Asymptotic decay of oscillatory solutions of second order differential equations with forcing term, Proc. Amer. Math. Soc., 66 (1977), pp. 251-257.

[21] T. Kusano - H. ONose, A nonoscillation theorem for a second order sublinear retarded differential equation, Bull. Austral. Math. Soc., 15 (1976), pp. 401-406.

[22] B. Srvgh, Forced nonoscillation in second order functional equations, Hiroshima Math. J., 7 (1977), pp. 657-665.

[23] P. W. SPIKEs, Some stability type results for a nonlinear differential equation, Rend. Mat., (6) 9 (1976), pp. 259-271.

[24] D. WILlett - J. S. W. Wong, Some properties of the solutions of $\left[p(t) x^{\prime}\right]^{\prime}+q(t) f(x)=0$, J. Math. Anal. Appl., 23 (1968), pp. 15-24.

[25] J. S. W. WONG, Remarles on stability conditions for the differential equation $x^{\prime \prime}+a(t) f(x)=0$, J. Austral. Math. Soc., 9 (1969), pp. 496-502.

[26] J. S. W. Wong, Some stability conditions for $x^{\prime \prime}+a(t) x^{2 n-1}=0$, SIAM J. Appl. Math., 15 (1967), pp. 889-892. 